\title{
Spinosin and 6"'-Feruloylspinosin protect the heart against acute myocardial ischemia and reperfusion in rats
}

\author{
MINHUA GU ${ }^{1,2}$, PEIKUN HE ${ }^{1}$, CHENFEI LYU ${ }^{3}$, XIAOYU LIU ${ }^{1}$, \\ YULING XU $^{1}$, SAIBO CHENG ${ }^{1}$, YONG GU ${ }^{3}$ and YUHUA JIA ${ }^{1}$ \\ ${ }^{1}$ School of Traditional Chinese Medicine, Southern Medical University, Guangzhou, Guangdong 510515; \\ ${ }^{2}$ Department of Chinese Medicine, Dongsheng Hospital, Guangzhou, Guangdong 510120; \\ ${ }^{3}$ Department of Neurology, Nanfang Hospital, Southern Medical University, Guangzhou, Guangdong 510515, P.R. China
}

Received February 1, 2019; Accepted August 30, 2019

DOI: $10.3892 / \mathrm{mmr} .2019 .10686$

\begin{abstract}
Investigating active compounds from Chinese herbal medicine that can rescue myocardial cells is a good approach to preserve cardiac function. Several herbal formulae that containing Semen Ziziphi Spinosae (SZS), also called Suanzaoren in Chinese, are clinically effective in the treatment of patients with acute myocardial infarction (AMI). The present study aimed to investigate the cardioprotective effects of spinosin and 6"'-feruloylspinosin, two flavonoid glycosides from SZS, in a rat model of myocardial ischemia and reperfusion. The left anterior descending artery (LAD) was occluded to induce myocardial ischemia. Spinosin or 6"'-feruloylspinosin $(5 \mathrm{mg} / \mathrm{kg})$ was intraperitoneally injected into rats $30 \mathrm{~min}$ before LAD ligation. The protein levels of myocardial enzymes in the serum, the extent of tissue injury and the rate of apoptosis were examined after AMI in rats with or without pretreatment with spinosin or 6 "'-feruloylspinosin. Western blotting was performed to investigate the potential mechanisms underlying the function of these two flavonoid glycosides. The present results suggested that pretreatment with spinosin or 6"'-feruloylspinosin significantly attenuated myocardial tissue injury, and reduced myocardial enzyme release and cell apoptosis in AMI rats. In addition, spinosin treatment increased light chain 3B-II and 6"'-feruloylspinosin,
\end{abstract}

Correspondence to: Professor Yuhua Jia, School of Traditional Chinese Medicine, Southern Medical University, 1838 Guangzhou Avenue North, Guangzhou, Guangdong 510515, P.R. China E-mail: yuhuajia_smu@126.com

Abbreviations: AMI, acute myocardial infarction; cTnI, cardiac troponin I; GSK3 $\beta$, glycogen synthase kinase- $3 \beta$; HE, hematoxylin and eosin; HO-1, hemeoxygenase 1; LAD, left anterior descending artery; LDH, lactate dehydrogenase; mPTP, mitochondrial permeability transition pore; Nrf2, nuclear factor (erythroid-derived 2)-like 2; PGC-1 $\alpha$, peroxisome proliferator-activated receptor $\gamma$ coactivator-1 $\alpha$; SOD, superoxide dismutase; SZS, Semen Ziziphi Spinosae; TUNEL, TdT-mediated dUTP Nick-End Labeling

Key words: AMI, SZS, spinosin, GSK3 $\beta$, autophagy and reduced p62, indicating that autophagy was promoted after drug treatments. Treatments of spinosin and 6"'-feruloylspinosin led to the reduction of glycogen synthase kinase-3 $\beta$ (GSK3 $\beta$ ) phosphorylation at Tyr216, and the increase of peroxisome proliferator-activated receptor $\gamma$ coactivator (PGC)-1 $\alpha$ and its downstream signaling proteins, including nuclear factor (erythroid-derived 2)-like 2 (Nrf2) and hemeoxygenase1 (HO-1). The present data suggested that SZS flavonoids could protect myocardial cells against acute heart ischemia-reperfusion, probably via the inhibition of GSK3 $\beta$, which increased autophagy and the activity of the PGC-1 $\alpha / \mathrm{Nrf} 2 / \mathrm{HO}-1$ pathway.

\section{Introduction}

Acute myocardial infarction (AMI) is one of leading causes of death and has become a major public health issue in both developed and developing countries (1). The aging population is increasing, and the AMI incidence rate is projected to increase (1). Early re-establishment of coronary reperfusion by thrombolytic therapy and endovascular intervention are the most effective strategies to reduce infarct size and preserve cardiac function (2). However, there are many patients with AMI who cannot benefit from coronary revascularization due to the time windows of thrombolysis and endovascular intervention, hemorrhagic tendency or reperfusion (2). Identifying cardioprotective reagents that can rescue injured myocardial cells is an important approach to preserve cardiac function.

Chinese herbal medicine has been used for thousands of years to treat diseases in human, and has recently attracted increasing attention in drug discovery research. Semen Ziziphi Spinosae (SZS), also called Suanzaoren in Chinese, is the seed of plant Ziziphus jujuba Mill. Spinosa $\mathrm{Hu}$ ex HF Chou, also known as jujube seeds. Generally, SZS is considered as a hypnotic-sedative herb and oriental medicine practitioners typically use it to treat insomnia and anxiety (3). Meanwhile, several formulations that containing SZS, such as Dingxin Recipe and Shensong Yangxin Capsule, were shown to exhibit cardioprotective effects and prevent ischemia/reperfusion-induced arrhythmias $(2,4)$. However, whether SZS or its components provide cardioprotective effects remains to be elucidated. 
SZS contains more than 50 bioactive compounds, including saponins, cyclopeptide alkaloids and C-glycoside flavonoids. Among them, 2"- $\beta$-O-glucopyranosyl swertisin (spinosin) and 6"'-feruloylspinosin (Fig. 1), two C-glycoside flavonoids, are used in healthcare products in Western countries and have been shown to exhibit pharmacological activities such as anxiolytic and hypnotic effects (5). In addition, spinosin was reported to increase neurogenesis, ameliorate memory deficit and enhance cognitive performance in mice $(6,7)$. A recent study suggested that spinosin exhibits neuroprotective effects in a mouse model of Alzheimer's disease (8). However, whether the two C-glycoside flavonoids have cardioprotective effects during ischemia-reperfusion injury remains to be elucidated.

The present study investigated the therapeutic potential of spinosin and 6"'-feruloylspinosin in a rat model of AMI. The cardioprotective effect of these two flavonoids and their underlying mechanisms were studied and compared.

\section{Materials and methods}

Experimental animals and ethics statement. Male Wistar albino rats (8-10 weeks) weighing 250-300 g were obtained from the Experimental Animal Center, Southern Medical University (Guangzhou, China). All animals were housed under a 12 -h light/dark cycle with an average temperature of $23^{\circ} \mathrm{C}$ and $40-60 \%$ humidity. Food and water were provided ad libitum. Randomization was performed by a third person unrelated to the study using a randomization table. All procedures were approved by the Ethic committee on Animal Experiments, Southern Medical University (approval no. L2017055) and followed the National Guidelines for Animal Experimentation (https://oacu.oir. nih.gov/animal-research-advisory-committee-guidelines).

Animals were acclimatized and fed until surgery. All efforts were made to minimize the number of animals used and their suffering.

Establishment of a rat model of AMI. Occlusion of the left anterior descending coronary artery (LAD) was employed to induce myocardial infarction to mimic human AMI, as previously described (9). Briefly, after being anesthetized by intraperitoneal injection of sodium pentobarbital $(50 \mathrm{mg} / \mathrm{kg}$, Merck $\mathrm{KGaA}$ ), the rats received mechanical ventilation with a volume-controlled rodent respirator (RWD Life Science Co., Ltd.). Then, the myocardial ischemia-reperfusion injury rat model was established by LAD ligation for $30 \mathrm{~min}$ followed by $1 \mathrm{~h}$ reperfusion. Rats were placed on a heating pad to maintain a temperature of $37^{\circ} \mathrm{C}$ during surgery. The rats were treated only with chest opening and threading but without LAD ligation. At the end of the reperfusion period, the animals were euthanized by intraperitoneal injection of $200 \mathrm{mg} / \mathrm{kg}$ sodium pentobarbital, and blood and heart tissues were collected for ELISA, hematoxylin and eosin (HE) or TUNEL staining, and western blot analysis.

Electrocardiogram and triphenyl tetrazolium chloride (TTC) staining were performed to verify if the AMI model had been successfully established. Before surgery onset, acupuncture needles were penetrated into the rat skin of four limbs, and then were connected to Pclab-530c biomedical information acquisition system (Pclab-530c; Beijing Microsignalstar) according to the manufacture's protocol. Standardized II lead was observed. For TTC staining, the heart tissue was placed into 1\% TTC (Sigma-Aldrich, St. Louis, MO) dissolved in 1X phosphate buffered saline in flask. After $10 \mathrm{~min}$ at $37^{\circ} \mathrm{C}$, the heart sections were imaged using a digital camera.

Drug administration. Animals were randomly assigned to four groups: i) Sham group; ii) vehicle group (Veh); iii) spinosin treated group (SP); and iv) 6"'-feruloylspinosin treated group (FS). Spinosin and 6"'-feruloylspinosin were dissolved in DMSO and further diluted in saline. Drugs were intraperitoneally administered $(5 \mathrm{mg} / \mathrm{kg}) 30 \mathrm{~min}$ before surgery. The dose and treatment methods were in accordance with two previous studies that focused on the effects of flavonoids on myocardial ischemia-reperfusion in rats $(10,11)$. The equal volume of DMSO-saline solution was used to treat AMI rats as vehicle control. A total of 48 rats were used in the study, with 12 rats in each group.

ELISA assay for determining the level of cardiac troponin I $(c T n I)$ in sera. After reperfusion, serum from each rat in four groups was collected and centrifuged for determining the level of cTnI by a commercial ELISA kit (JLC2389, Shanghai Jiang Kang Biotech) according to the manufacturer's instructions.

Evaluation of lactate dehydrogenase $(\mathrm{LDH})$ activity. Sera were also used to detect LDH activity. $\mathrm{LDH}$ catalyzes lactic acid to produce pyruvate which reacts with 2,4-dinitrophenylhydrazine to yield pyruvate dinitrophenylhydrazone, exhibiting a brown color in alkaline solution and which can be quantified by colorimetric analysis (12). A commercial kit was purchased to detect LDH activity (Nanjing Jiancheng Bioengineering Institute) in accordance the manufacturer's instructions. The absorbance was measured at $450 \mathrm{~nm}$ using a microplate reader (Bio-Rad Laboratories, Inc.).

HE staining of histological sections. The heart tissues were isolated to observe the integrity and injury in different groups by $\mathrm{HE}$ staining. Briefly, after ischemia and reperfusion, rat hearts were isolated and the heart tissues were fixed in $4 \%$ paraformaldehyde overnight at room temperature, and then dehydrated by passing through gradient concentrations of ethanol $(80 \%$ for $2 \mathrm{~h}, 90 \%$ for $2 \mathrm{~h}, 95 \%$ overnight, $100 \%$ for $0.5,0.5$ and $1 \mathrm{~h})$ at room temperature, followed by embedding in paraffin wax. The paraffin-embedded samples were then sectioned at $4 \mu \mathrm{m}$ for staining with Mayer's Hematoxylin (H8070, Solarbio Life Sciences, Beijing, China) for $10 \mathrm{~min}$ and then by $0.5 \%$ aqueous eosin (DH0050, Leigene Biotech, Beijing, China) for $3 \mathrm{~min}$ at room temperature. With this method, nucleus and other acidic structures are stained blue, while cytoplasm is stained red. Images were acquired using a light microscope at x50 or x400 magnification (Leica Microsystems $\mathrm{GmbH}$ ).

TUNEL staining on heart specimen. Rat heart tissues from various groups were collected to determine cell apoptosis using DeadEnd Fluorometric TUNEL System (Promega Corporation). A portion of heart tissues were fixed in $4 \%$ paraformaldehyde overnight at room temperature and then sectioned at $10 \mu \mathrm{m}$ thickness for TUNEL staining of fragmented DNA 
A<smiles>COc1cc2oc(-c3ccc(O)cc3)cc(=O)c2c(O)c1[C@@H]1O[C@H](CO)[C@@H](O)[C@H](O)[C@H]1O[C@@H]1O[C@H](CO)[C@@H](O)[C@H](O)[C@H]1O</smiles><smiles>COc1cc(/C=C/C(=O)OC[C@H]2O[C@H](O[C@H]3[C@H](c4c(OC)cc5oc(-c6ccc(O)cc6)cc(=O)c5c4O)O[C@H](CO)[C@@H](O)[C@@H]3O)[C@H](O)[C@@H](O)[C@@H]2O)ccc1O</smiles>

Figure 1. The chemical structures of the flavonoids examined. (A) Spinosin. (B) 6"'-feruloylspinosin.

according to manufacturer's instructions. The stained specimen was then mounted using Fluoroshield Mounting Medium with DAPI (Abcam) to stain all nuclei, followed by observation under a fluorescent microscope (Olympus Corporation). Then, three sections of each heart were observed, and the average TUNEL-positive cells were counted using ImageJ $1.48 \mathrm{v}$ (National Institutes of Health), to the maximum extent possible, to keep results uniform and accurate. The total number of positive nuclei was determined by counting all the labeled nuclei present in the entire specimen.

Western blot analysis. Heart tissues of the same regions were homogenized in RIPA lysis buffer (Leigen Biotech Co. Ltd.) containing protease and phosphatase inhibitors (MedChemExpress). Total proteins of each sample were quantified by a BCA quantification kit (FD2001, Fdbio Science). The expression of each protein in these samples was analyzed by Western blot, according to a previous literature (13). In brief, after being denatured in loading buffer, a total of $30 \mu \mathrm{g}$ proteins in each sample were separated by SDS-PAGE on $8-10 \%$ gels and then transferred to polyvinylidene difluoride membranes (EDM Millipore). Following blocking with 5\% non-fat milk for $1 \mathrm{~h}$ at room temperature, the membranes were incubated overnight at $4^{\circ} \mathrm{C}$ with primary antibodies including anti-light chain (LC)3B (1:1,000, cat. no. ab48394; Abcam), anti-p62 (1:1,000, cat. no. ab56416; Abcam), anti-phosphorylated glycogen synthase kinase-3 $\beta(1: 1,000$, pGSK3 $\beta$; cat. no. ab75745; Abcam), anti-GSK3 $\beta$ (1:1,000, cat. no. ab131356; Abcam), anti-nuclear factor (erythroid-derived 2)-like 2 (Nrf2; 1:1,000, cat. no. 16396-1-AP; ProteinTech Group, Inc.), anti-heme oxygenase 1 (HO-1; 1:1,000, cat. no. ab13243; Abcam), anti-PGC-1 $\alpha$ (peroxisome proliferator-activated receptor $\gamma$ coactivator $1 \alpha ; 1: 1,000$, cat. no. ab54481; Abcam), $\beta$-actin (1:5,000, Santa Cruz Biotechnology, Inc.), GAPDH (1:10,000, cat. no. 10494-1-AP; ProteinTech Group, Inc.) and SOD-2 (1:1,500, cat. no. sc-137254; Santa Cruz Biotechnology, Inc.), followed by incubation with anti-rabbit $(1: 5,000$, SA00001-2, ProteinTech Group, Inc.) or anti-mouse (1:5,000, cat. no. sc-2005, Santa Cruz Biotechnology, Inc.) horseradish peroxidase-conjugated secondary antibodies. Bands were detected by chemiluminescence technology using enhanced chemiluminescence advance Western blotting detection reagents (FD8020, Fdbio Science), quantified and normalized to $\beta$-actin or GAPDH using ImageJ $1.48 \mathrm{v}$ (National Institutes of Health) by an experimenter blinded to group allocation.

Statistical analysis. Data were expressed as the mean \pm SD. Differences within multiple groups were examined by two-way ANOVA followed by the Bonferroni post hoc test. Statistical analyses were conducted using SPSS 20.0 (IBM Corp.) and GraphPad Prism 5.0 (GraphPad Software, Inc.). P $<0.05$ was considered to indicate a statistically significant difference. 
A

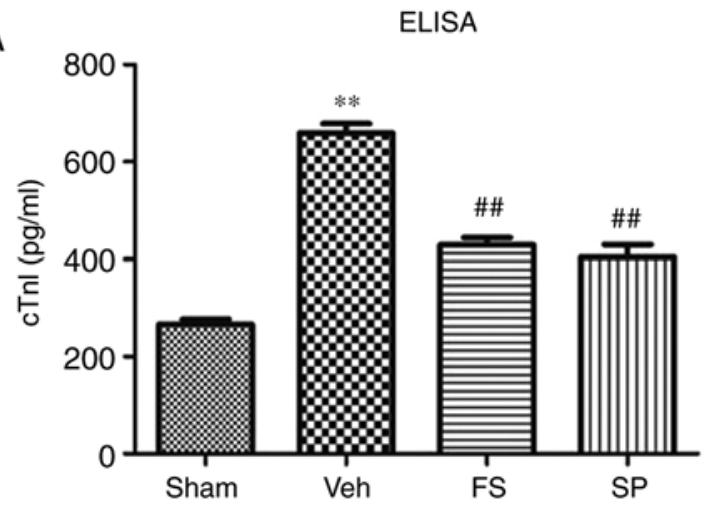

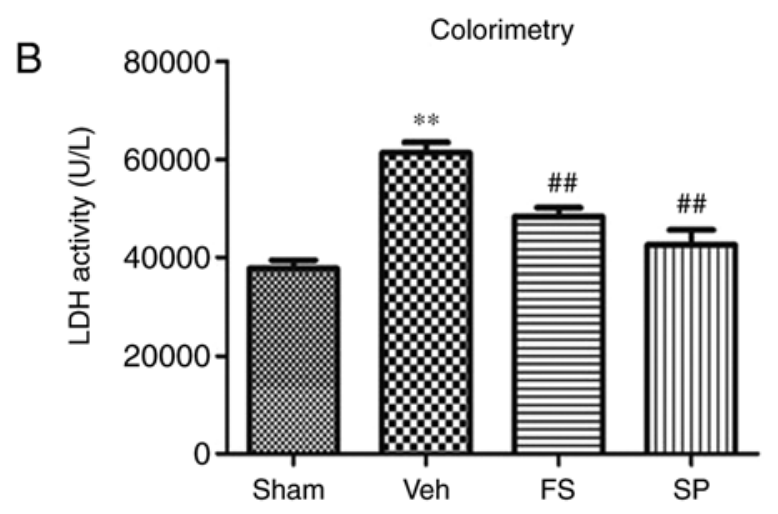

Figure 2. SP and FS decreased serum cTnI level and LDH activity in AMI rats. (A) Sera from rats in Sham, Veh-, SP- and FS-treated AMI model rats were collected and subjected to ELISA analysis for determining cTnI concentrations. Data demonstrated that SP and FS significantly reduced serum cTnI level. (B) LDH activity was determined with a commercial kit and results revealed SP and FS could decrease LDH activity in rats with myocardial infarction. Data were expressed as the mean \pm standard deviation; $n=6$ in each group; ${ }^{* *} \mathrm{P}<0.01$ vs. Sham; ${ }^{\# \#} \mathrm{P}<0.01$ vs. Veh. SP, Spinosin; FS, $6 "$ '-feruloylspinosin; cTnI, cardiac troponin I; LDH, lactate dehydrogenase; AMI, acute myocardial infarction; Veh, vehicle.

\section{Results}

Spinosin or 6"'-feruloylspinosin treatment reduces cardiac troponin level and $\mathrm{LDH}$ activity in sera from AMI rats. To explore the cardioprotective effects of spinosin and 6"'-feruloylspinosin, a rat model of AMI and reperfusion was constructed. TTC staining and electrocardiogram were used to monitor and confirm the success of myocardial infarction model (Fig. S1). The present results suggested that myocardial infarction induced notable increases in cardiac troponin I (cTnI) level and LDH activity $(\mathrm{P}<0.01$; Fig. 2$)$. In addition, both spinosin and 6"'-feruloylspinosin could significantly reduce the serum levels of cTnI (from 662.0 to 402.7 and $429.2 \mathrm{pg} / \mathrm{ml}$, respectively; $\mathrm{P}<0.01$; Fig. $2 \mathrm{~A})$ and the activities of $\mathrm{LDH}(61286$ to 42516 and $48270 \mathrm{U} / \mathrm{ml}$, respectively; $\mathrm{P}<0.01$; Fig. $2 \mathrm{~B}$ ) in AMI model rats.

ZSC Flavonoids treatment reduces heart tissue injury and myocardial cell apoptosis in AMI rats. Next, following ischemia-reperfusion, the hearts were used to compare the tissue injury and cell apoptosis in different groups. HE staining results demonstrated that myocardial cells from sham operative rats were regularly arranged, associating with intact muscle fibers, while no cell edema or cell death was observed (Fig. 3A). In AMI rats treated with vehicle, however, some myocardial cells presented edema and death, accompanied by local hemorrhage and neutrophil infiltration (Fig. 3B). These pathological features were attenuated after treatment with spinosin or 6"'-feruloylspinosin (Fig. 3C and D).

Whether the regulation of cell apoptosis program participated in the cardioprotective effects was subsequently examined. As shown in Fig. 4, 55.3\% apoptotic myocardial cells were observed in vehicle treated AMI rats, and the rate was reduced to 39.0 and $38.1 \%$ after treatment by spinosin and 6 "'-feruloylspinosin, separately $(\mathrm{P}<0.01$; Fig. 4B). Taken together, the present results suggested that flavonoids in ZSC could protect heart against acute ischemia-reperfusion damage.

Spinosin or 6"'-feruloylspinosin treatment can promote cell autophagy and reduce the phosphorylation of GSK3 $\beta$ in AMI rats. Pharmacological modulation of autophagy has been shown to affect disease outcome in a rodent model of myocardial infarction (14). Therefore, in the present study, the autophagy markers and relevant regulatory molecules were examined in various experimental groups. The present results suggested that spinosin and 6"'-feruloylspinosin increased the level of LC3B-II, while 6"'-feruloylspinosin could decrease p62, compared with vehicle control, determined by western blot analysis of infarct myocardial tissues (Fig. 5A and B), indicating they may promote cell autophagy of myocardial cells in AMI rats.

Inhibiting the phosphorylation of GSK $3 \beta$ at Tyrosin 216 can significantly increase the basal autophagy via AMP activated protein kinase-dependent pathway (15). pGSK3 $\beta$ and total GSK3 $\beta$ were then immune-blotted with specific antibodies, and phosphorylation of GSK3 $\beta$ was increased up to 6 times compared with the sham control $(\mathrm{P}<0.01)$, and decreased after treatment of spinosin or 6"'-feruloylspinosin ( $\mathrm{P}<0.01$; Fig. $5 \mathrm{C})$.

Spinosin or 6"'-feruloylspinosin treatment enhances the expressions of $\mathrm{PGC}-1 \alpha, \mathrm{Nrf} 2$ and $\mathrm{HO}-1$ in AMI rats. Evidence has shown the importance of GSK3 $\beta$ in ischemia-reperfusion pathologies of heart, not only effects autophagy, but also regulates multiple downstream cell-protective signal molecules, including PGC-1 $\alpha$, Nrf2, HO-1 and SOD2 (16). Therefore, the expressions of these molecules in samples from different groups were detected. As shown in Fig. 6, the expressions of Nrf 2 and PGC-1 $\alpha$ were decreased in the AMI model group compared with the sham group, and clearly increased by spinosin or 6"'-feruloylspinosin treatment (Fig. 6A and B). The expression of HO-1 was also significantly induced (Fig. 6C). Notably, 6"'-feruloylspinosin had higher efficiency to induce the expressions of PGC-1 $\alpha$, Nrf2 and HO-1. However, SOD-2 expression exhibited no obvious change after drug treatment (Fig. 6D).

\section{Discussion}

To the best of our knowledge, this is the first study to report that spinosin and 6"'-feruloylspinosin, two C-glycoside flavonoids 

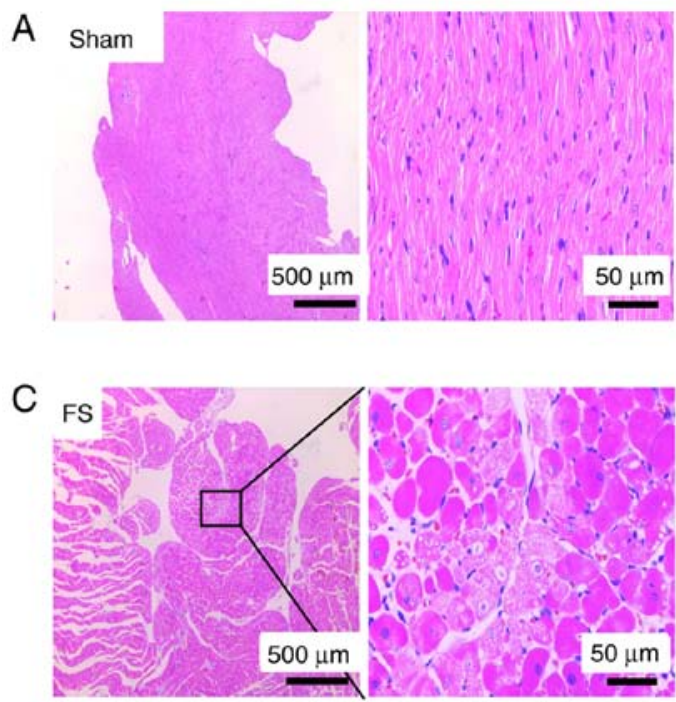

B

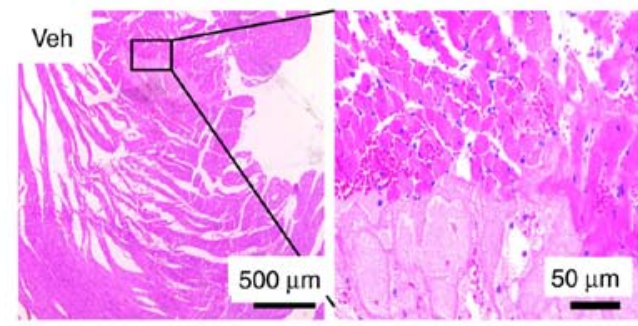

D

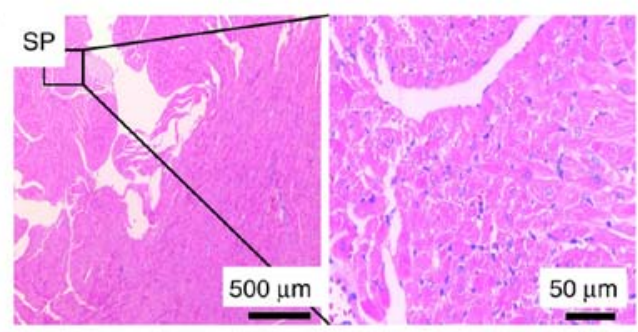

Figure 3. HE staining assay showing SP and FS ameliorated tissue injury in hearts isolated from rats with myocardial infarction. (A) HE staining graphs in Sham group. Myocardial cells were regular and neatly arranged, muscle fibers were intact and no cell edema or death was observed. (B) HE staining picture in Veh-treated AMI rats. Some myocardial cells demonstrated edema and death, accompanied by local hemorrhage and neutrophil infiltration. HE staining in (C) SP and (D) FS treated AMI rats. The above pathological features were attenuated after drug treatments. Graphs on right side are the amplifications of the left picture. Scale bar is $500 \mu \mathrm{m}$ on left, and $50 \mu \mathrm{m}$ on right. $\mathrm{n}=6$ in each group. HE, hematoxylin-eosin; SP, Spinosin; FS, 6"'-feruloylspinosin; AMI, acute myocardial infarction; Veh, vehicle.

A
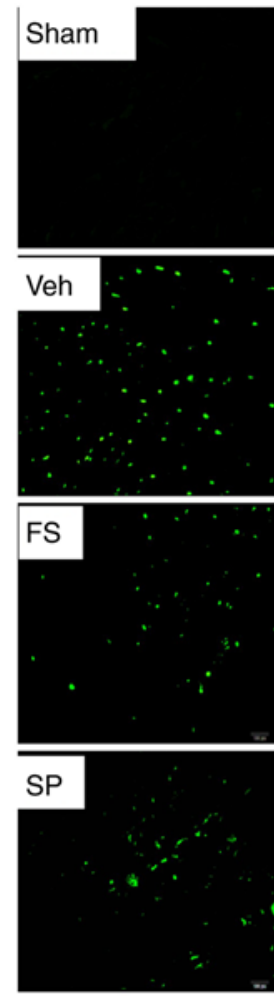

TUNEL
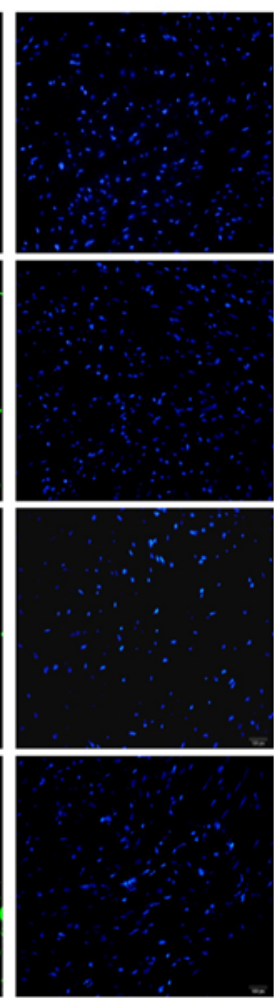

DAPI
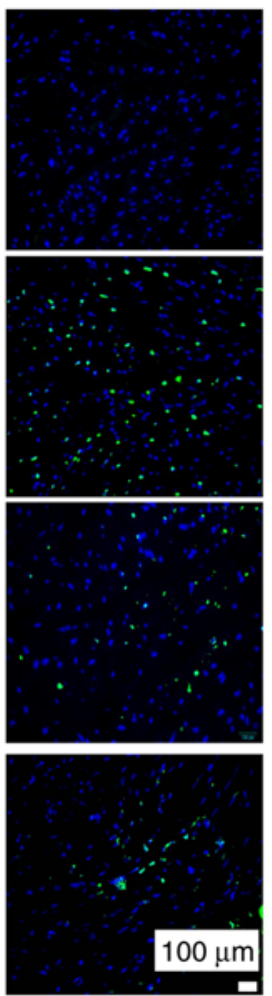

Merge
B

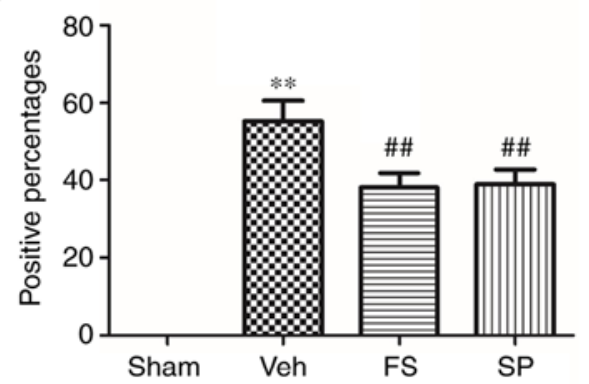

Figure 4. TUNEL assay revealing SP and FS decreased apoptotic cell death in the heart after myocardial infarction. (A) TUNEL staining images and nuclei staining in each group. (B) Quantification of the percentages of positive staining cells in different groups. Scale bar is $100 \mu \mathrm{m}$. Data were expressed as mean \pm standard deviation; $n=6$ in each group; ${ }^{* *} \mathrm{P}<0.01$ vs. Sham; ${ }^{\# \#} \mathrm{P}<0.01$ vs. Veh. SP, Spinosin; FS, 6"'-feruloylspinosin; Veh, vehicle.

from SZS, could protect the heart against ischemia-reperfusion injury in a rat model AMI. It was found that they could significantly attenuate myocardial tissue injury and reduce myocardial enzyme release and cell apoptosis. In addition, they could promote autophagy flux and increase the levels of PGC1 $\alpha$, Nrf2 and HO-1, which may be related to the reduction of phosphorylation of GSK3 $\beta$. The present study not only improves the current understanding of the cardioprotective 
A

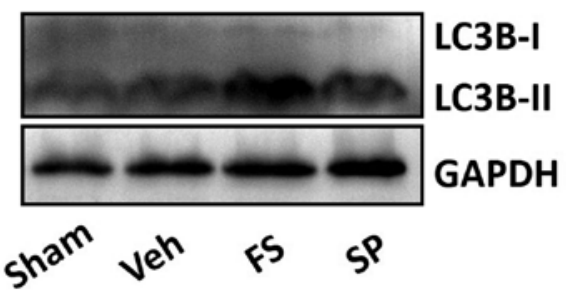

B

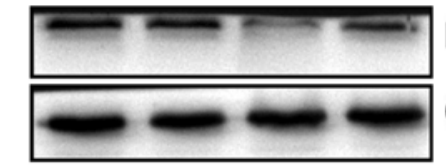

p62

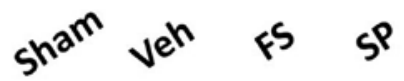

C
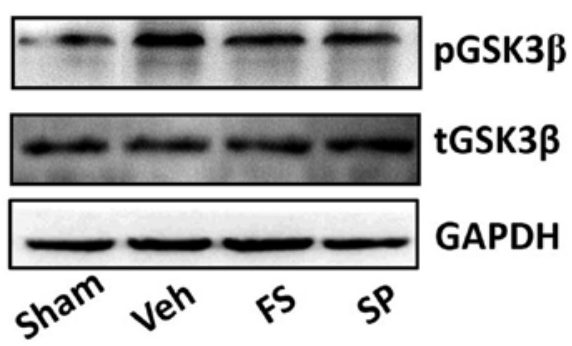

tGSK3 $\beta$

GAPDH

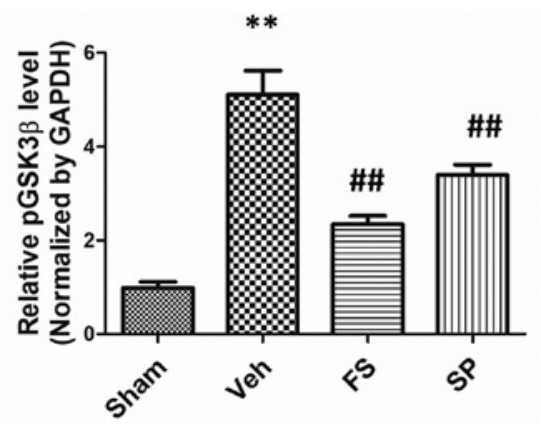

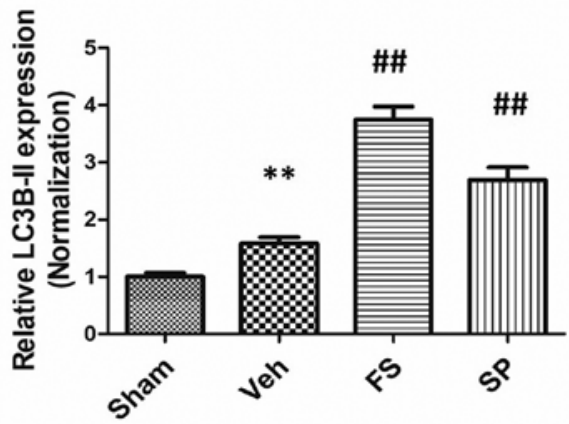
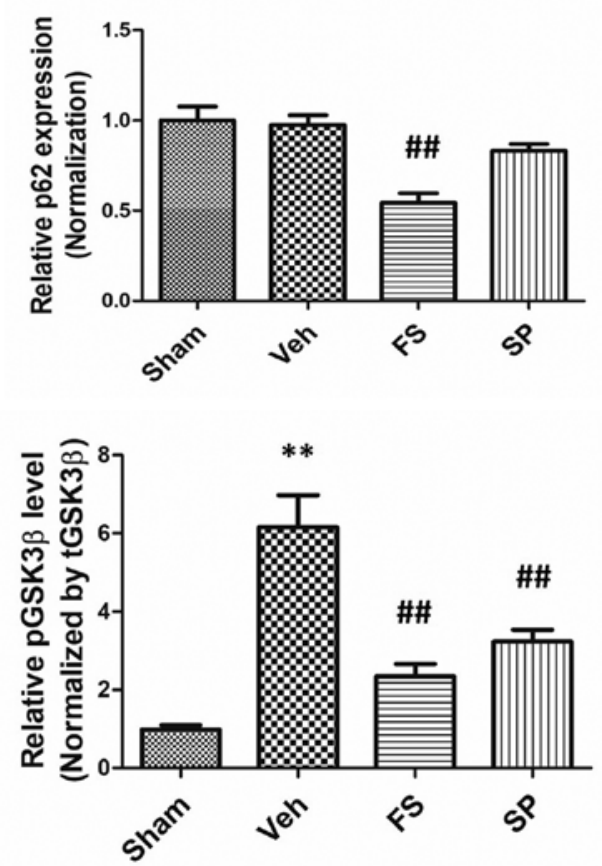

n.s.

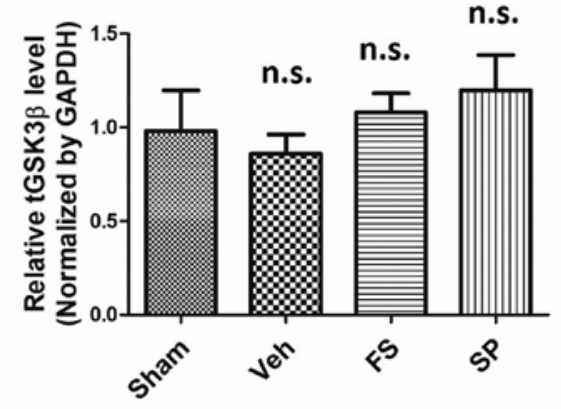

Figure 5. Western blot analyses for detecting the levels of markers (LC3B and p62), as well as an important signal molecule (pGSK3 $\beta$ at Tyr216), of autophagy. Representative Western blot results in each group showing the relative levels of LC3B (A), p62 (B) and pGSK3 $\beta$ (C). Quantification of band intensities were normalized to GAPDH and/or total GSK3 $\beta$ expressed as bar graph. Results demonstrated that SP and FS increased the level of LC3B-II and decreased the level of pGSK3 $\beta$, indicating SP and FS promotes autophagy flux. Data were expressed as mean \pm standard deviation; $\mathrm{n}=6$ in each group; $\mathrm{n} . \mathrm{s}$. not significant; ${ }^{* *} \mathrm{P}<0.01$ vs. Sham; ${ }^{\# \#} \mathrm{P}<0.01$ vs. Veh. LC, light chain; pGSK3 $\beta$, phosphorylated glycogen synthase kinase-3 $\beta$; SP, Spinosin; FS, 6 "'-feruloylspinosin; Veh, vehicle; t-, total.

mechanisms of spinosin and 6"'-feruloylspinosin, but also advances the current knowledge about the active ingredients of SZS with cardioprotective effects.

Previous studies have focused on the anti-anxiety and sedative effects of spinosin, probably because SZS is defined as a hypnotic-sedative herb and is usually used for the treatment of insomnia and anxiety in East Asia countries.
Recently, anti-dementia, neuroprotective and neurogenesis effects have been identified by several independent groups (6-8). Considering the protection of neurons and myocardial cells usually share common mechanism, it is not surprising that, in present study, spinosin protected the heart against ischemia-reperfusion injury in AMI rats, especially when cardioprotective roles of many other herbal flavonoids 
A

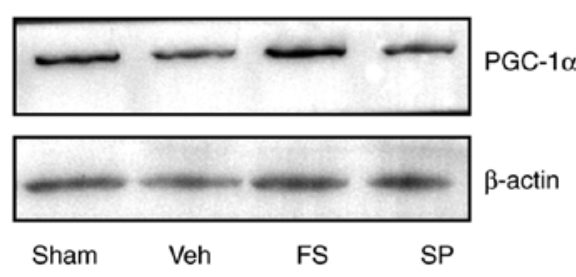

B

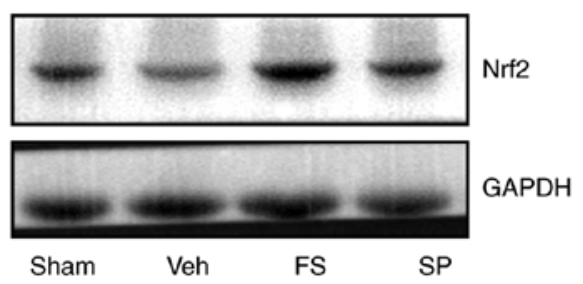

C

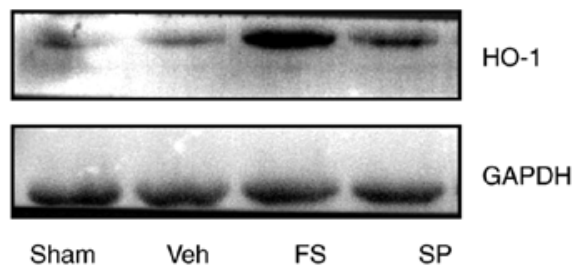

D
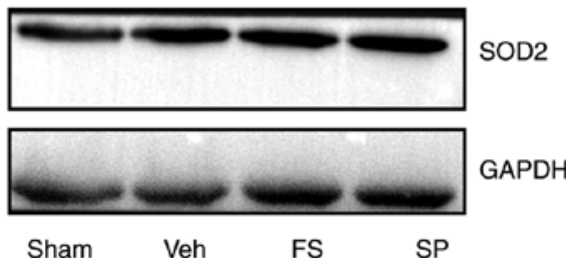
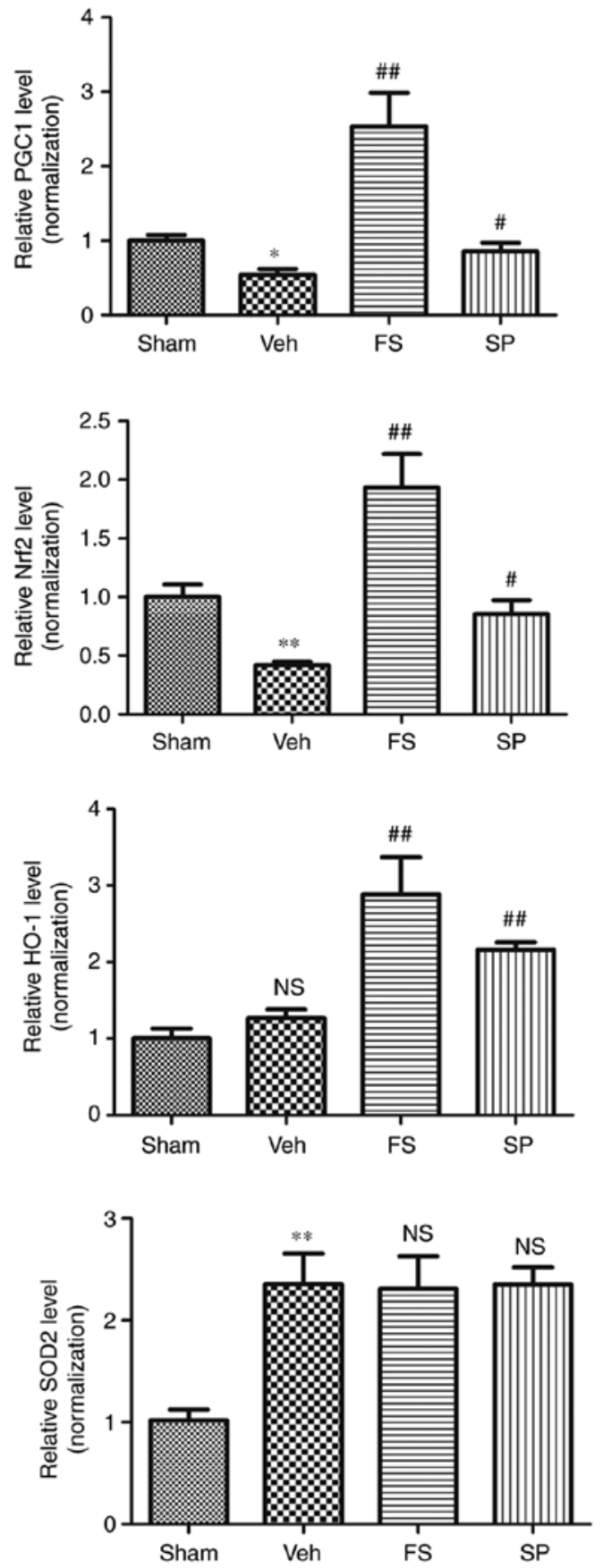

Figure 6. Western blot analysis for determining the expressions of PGC-1 $\alpha$, Nrf2, HO-1 and SOD-2, downstream molecules of pGSK3 $\beta$. Representative western blot results in each group showing the relative levels of (A) PGC-1 $\alpha$, (B) Nrf2, (C) HO-1 and (D) SOD2. Quantification of band intensities were expressed as bar graph and shown on right side. Data indicated SP and FS could increase the expressions of PGC-1, Nrf2 and HO-1, but not SOD2. Data were expressed as Mean \pm standard deviation; $\mathrm{n}=6$ in each group; $\mathrm{n} . \mathrm{s}$. not significant; ${ }^{*} \mathrm{P}<0.05,{ }^{* *} \mathrm{P}<0.01$ vs. Sham; ${ }^{\#} \mathrm{P}<0.05,{ }^{\# \#} \mathrm{P}<0.01$ vs. Veh. $\mathrm{PGC}-1 \alpha$, peroxisome proliferator-activated receptor $\gamma$ coactivator-1 $\alpha$; Nrf2, nuclear factor (erythroid-derived 2)-like 2; HO-1, hemeoxygenase 1; SOD, superoxide dismutase; pGSK3 $\beta$, phosphorylated glycogen synthase kinase-3 $\beta$; SP, Spinosin; FS, 6"'-feruloylspinosin; Veh, vehicle.

are taken into consideration (17). However, the present study extended the pharmacological view on the active ingredients in SZS and provided a potential for drug discovery in cardiovascular diseases.

Myocardial ischemia-reperfusion injury is a complicated pathophysiological process in which oxidative stress, calcium overload, inflammatory responses and myocardial apoptosis serve important roles $(1,18)$. An excessive generation of reactive oxygen species (ROS) or free radicals, mostly in mitochondria, is a trigger factor of injury during reperfusion stage.
The elevation of ROS can trigger opening of mitochondrial permeability transition pore (mPTP), which in turn amplifies ROS production (so called ROS-induced ROS production), resulting in detrimental consequences for the affected myocardial cells (19). Approaches, such as inhibition of GSK3 $\beta$, that restrict $\mathrm{mPTP}$ induction by increasing the mPTP-ROS threshold can provide myocardial cell protection (16). In fact, GSK3 $\beta$ has been proved to mediate convergence of myocardial protection $(16,20)$. Signal pathways and agents revolving around GSK $3 \beta$ are intensively investigated to explore the 
mechanisms and approaches. The present study provided evidence showing spinosin and 6"'-feruloylspinosin reduced the phosphorylation of GSK $3 \beta$ at Tyr216, and probably thereby protected myocardial cells. Fully elucidating the mechanism requires the comparison between SZS flavonoids and specific GSK3 $\beta$ inhibitors.

Inhibition of GSK $3 \beta$ not only directly controls mPTP through interacting mitochondrial proteins, but also induces multiple cell survival pathways, including prosurvival autophagy (21) and PGC-1 $\alpha / \mathrm{Nrf} 2 / \mathrm{HO}-1$ (22). Autophagy is a catabolic process to degrade cellular long-lived proteins and components in lysosome, while the metabolites then can be reused as the source of energy for the synthesis of new macromolecules $(23,24)$. Autophagy plays a critical role in myocardial survival following AMI. Natural compounds, such as berberine and salvianolic acid B, protect myocardial tissue against ischemia-reperfusion by promoting autophagy $(25,26)$. GSK $3 \beta$ inhibition also promotes autophagy to protect myocardial cells (21). Following this evidence, the present study identified that both spinosin and 6"'-feruloylspinosin could enhance the expression of LC3B-II, while 6"'-feruloylspinosin could reduce the level of p62, indicating autophagy is activated and probably involved in cardioprotection in the AMI model. Enhanced level of LC3B-II and reduced p62 represent more cells undergoing autophagy flux. During autophagy procedures, target engulfment and sequestration depend on p62 which is degraded by autophagy after binding to LC3 during autophagosome formation and is accepted as an autophagic substrate (27). However, it is not completely clear if p62 is exclusively eliminated by autophagy or if the ubiquitin-proteasome pathway could also be involved (28). In the present study, spinosin and 6"'-feruloylspinosin could enhance the expression of LC3B-II, while only 6 "'-feruloylspinosin could reduce the level of p62, indicating other different mechanisms might be involved in regulating autophagy flux.

PGC-1 $\alpha$ is an inducible transcription co-activator that is involved in the repair of ROS-induced mitochondrial injury, biogenesis of mitochondria and regulation of energy metabolism $(22,29)$. GSK $3 \beta$ can phosphorylate PGC- $1 \alpha$ and promote its ubiquitin-dependent degradation, whereas GSK $3 \beta$ inhibition can induce the accumulation of PGC-1 $\alpha$ to protect cells $(30,31)$. The cytoprotective effect of PGC-1 $\alpha$ is related to the upregulation of Nrf2, a transcription factor, followed by the transcriptional activation of anti-oxidant gene HO-1 (22), an enzyme catalyzes the degradation of pro-oxidant heme into three important antioxidant products, bilirubin, carbon monoxide and ferrous ion (32). Meanwhile, Nrf2 can also induce the expression of SOD-2. In agreement with their inhibitory roles on GSK3 $\beta$, spinosin and 6"'-feruloylspinosin upregulated the protein levels of PGC- $1 \alpha$, Nrf 2 and HO-1, indicating GSK3 $\beta$ inhibition-mediated PGC- $1 \alpha / \mathrm{Nrf} 2 / \mathrm{HO}-1$ participates in the cardioprotective roles of SZS flavonoids. Changes in SOD-2 were not observed following flavonoids treatment, so presumably other unknown regulators may be involved.

Data from the present study demonstrated that 6 "'-feruloylspinosin had a higher efficiency on the activation of PGC-1 $\alpha / \mathrm{Nrf} 2 / \mathrm{HO}-1$ pathway than spinosin, although they did not have significant differences in cardioprotective effects. The distinction on regulating cell protective molecules is possibly due to the different pharmacokinetic properties. A previous study revealed that 6 "'-feruloylspinosin was distributed in rats faster, and was more slowly eliminated from the plasma than spinosin, with a half-life $2.25 \pm 0.52 \mathrm{~h}$ of 6 "'-feruloylspinosin, compared with the $1.39 \pm 0.11 \mathrm{~h}$ of spinosin (33). However, carefully comparing the protective efficiency of spinosin and 6 "'-feruloylspinosin requires multiple methods and dosages of drugs administration.

Finally, SZS-containing formulae are a commonly used herbal medicine for the treatment of patients with AMI and stenocardia in Traditional Chinese Medicine, and C-glycoside flavonoids are active compounds isolated from SZS. The findings of the present study can be used to understand the cardioprotective mechanisms of SZS. It is notable that formulae in Chinese Medicine contains multiple active compounds and other active compounds may also contribute to the protection of heart against myocardial ischemia-reperfusion injury. More attention should be paid to the synergetic actions of the active compounds in the medicinal herb against myocardial ischemia-reperfusion injury in future.

There are several limitations to the present study. First, cytotoxicity tests of the two drugs should be performed before an effectiveness study to ensure their safety. Secondly, myocardial ischemia-reperfusion group rats without any treatment were not added, and therefore, the effect of myocardial ischemia-reperfusion itself on the tissue injury and enzyme release was not observed. Third, the molecular mechanisms of the cardioprotective effects of spinosin and 6"'-feruloylspinosin remain unclear, and it is planned to carefully investigate how the PGC- $1 \alpha / \mathrm{Nrf} 2 / \mathrm{HO}-1$ pathway is involved in the protective role during which the inhibitor and genetic deletion of PGC-1 $\alpha$ would be employed.

\section{Acknowledgements}

Not applicable.

\section{Funding}

The present study was supported by the Science and Technology Planning Project of Guangdong Province of China (grant no. 2016A020226002), the Major Projects of Industry-University-Research Collaboration Innovation, the Science and Technology Program of Guangzhou, China (grant no. 201704020042), and the Second Development of Famous Chinese Patent Medicine of Guangdong Province, China (grant no. 20174010).

\section{Availability of data and materials}

The datasets used and/or analyzed during the current study are available from the corresponding author on reasonable request.

\section{Authors' contributions}

MG, PH, CL, XL, YX and SC conducted the experiments. MG and SC performed statistical analysis. YG and YJ designed the study and analyzed the data. MG, YG, and YJ wrote the manuscript. YJ gave final approval of the publication. All authors read and approved the final manuscript. 


\section{Ethics approval and consent to participate}

All animal operations were approved by the Ethic committee on Animal Experiments, Southern Medical University (approval no. L2017055) and followed the National Guidelines for Animal Experimentation.

\section{Patient consent for publication}

Not applicable.

\section{Competing interests}

The authors declare that they have no competing interests.

\section{References}

1. Frank A, Bonney M, Bonney S, Weitzel L, Koeppen M and Eckle T: Myocardial ischemia reperfusion injury: From basic science to clinical bedside. Semin Cardiothorac Vasc Anesth 16 123-132, 2012.

2. Binder A, Ali A, Chawla R, Aziz HA, Abbate A and Jovin IS Myocardial protection from ischemia-reperfusion injury post coronary revascularization. Expert Rev Cardiovasc Ther 13 1045-1057, 2015

3. Cao JX, Zhang QY, Cui SY, Cui XY, Zhang J, Zhang YH, Bai YJ and Zhao YY: Hypnotic effect of jujubosides from Semen Ziziph Spinosae. J Ethnopharmacol 130: 163-166, 2010.

4. Jia YH, Zhang YX, Li LJ, Liu YW, Li CH, Fu XQ, Zeng P, $\mathrm{Wu}$ WK and Sun XG: Dingxin recipe prevents ischemia/reperfusion-induced arrhythmias via up-regulating prohibitin and suppressing inflammatory responses. Chin J Integr Med 18 120-129, 2012

5. Liu J, Zhai WM, Yang YX, Shi JL, Liu QT, Liu GL, Fang N, Li J and Guo JY: GABA and 5-HT systems are implicated in the anxiolytic-like effect of spinosin in mice. Pharmacol Biochem Behav 128: 41-49, 2015.

6. Lee Y, Jeon SJ, Lee HE, Jung IH, Jo YW, Lee S, Cheong JH, Jang DS and Ryu JH: Spinosin, a C-glycoside flavonoid, enhances cognitive performance and adult hippocampal neurogenesis in mice. Pharmacol Biochem Behav 145: 9-16, 2016.

7. Ko SY, Lee HE, Park SJ, Jeon SJ, Kim B, Gao Q, Jang DS and Ryu JH: Spinosin, a C-Glucosylflavone, from Zizyphus jujuba var. Spinosa ameliorates abeta1-42 oligomer-induced memory impairment in Mice. Biomol Ther (Seoul) 23: 156-164, 2015.

8. Xu F, He B, Xiao F, Yan T, Bi K, Jia Y and Wang Z: Neuroprotective effects of spinosin on recovery of learning and memory in a mouse model of Alzheimer's disease. Biomol The (Seoul) 27: 71-77, 2019

9. Wang J, Yang H, Hu X, Fu W, Xie J, Zhou X, Xu W and Jiang H: Dobutamine-mediated heme oxygenase-1 induction via PI3K and p38 MAPK inhibits high mobility group box 1 protein release and attenuates rat myocardial ischemia/reperfusion injury in vivo. J Surg Res 183: 509-516, 2013.

10. Guo X, Cao W, Yao J, Yuan Y, Hong Y, Wang X and Xing J: Cardioprotective effects of tilianin in rat myocardial ischemia-reperfusion injury. Mol Med Rep 11: 2227-2233, 2015.

11. Colareda GA and Consolini AE: Low-flow ischaemia and reperfusion in rat hearts: Energetic of stunning and cardioprotection of genistein. J Pharm Pharmacol 70: 1174-1187, 2018.

12. Dou MM, Zhang ZH, Li ZB, Zhang J and Zhao XY: Cardioprotective potential of Dendrobium officinale Kimura et Migo against myocardial ischemia in mice. Mol Med Rep 14 4407-4414, 2016

13. Djavaheri-Mergny M, Amelotti M, Mathieu J, Besançon F, Bauvy $C$, Souquère $S$, Pierron $G$ and Codogno P: NF-kappaB activation represses tumor necrosis factor-alpha-induced autophagy. J Biol Chem 281: 30373-30382, 2006.

14. Bravo-San Pedro JM, Kroemer G and Galluzzi L: Autophagy and mitophagy in cardiovascular disease. Circ Res 120: 1812-1824, 2017
15. Weikel KA, Cacicedo JM, Ruderman NB and Ido Y: Knockdown of GSK3 $\beta$ increases basal autophagy and AMPK signalling in nutrient-laden human aortic endothelial cells. Biosci Rep 36: pii: $\mathrm{e} 00382,2016$

16. Juhaszova M, Zorov DB, Yaniv Y, Nuss HB, Wang S and Sollott SJ: Role of glycogen synthase kinase-3beta in cardioprotection. Circ Res 104: 1240-1252, 2009.

17. Romano B, Pagano E, Montanaro V, Fortunato AL, Milic N and Borrelli F: Novel insights into the pharmacology of flavonoids. Phytother Res 27: 1588-1596, 2013.

18. Sinning C, Westermann D and Clemmensen P: Oxidative stress in ischemia and reperfusion: Current concepts, novel ideas and future perspectives. Biomark Med 11: 11031-11040, 2017.

19. Zorov DB, Juhaszova $M$ and Sollott SJ: Mitochondrial ROS-induced ROS release: An update and review. Biochim Biophys Acta 1757: 509-517, 2006.

20. Ghaderi S, Alidadiani N, Dilaver N, Heidari HR, Parvizi R, Rahbarghazi R, Soleimani-Rad J and Baradaran B: Role of glycogen synthase kinase following myocardial infarction and ischemia-reperfusion. Apoptosis 22: 887-897, 2017.

21. Marchand B, Arsenault D, Raymond-Fleury A, Boisvert FM and Boucher MJ: Glycogen synthase kinase-3 (GSK3) inhibition induces prosurvival autophagic signals in human pancreatic cancer cells. J Biol Chem 290: 5592-5605, 2015.

22. Choi HI, Kim HJ, Park JS, Kim IJ, Bae EH, Ma SK and Kim SW: PGC-1 $\alpha$ attenuates hydrogen peroxide-induced apoptotic cell death by upregulating Nrf-2 via GSK3 $\beta$ inactivation mediated by activated p38 in HK-2 Cells. Sci Rep 7: 4319, 2017.

23. Boya P, Reggiori F and Codogno P: Emerging regulation and functions of autophagy. Nat Cell Biol 15: 713-720, 2013

24. Rabinowitz JD and White E: Autophagy and metabolism. Science 330: 1344-1348, 2010.

25. Zhang YJ, Yang SH, Li MH, Iqbal J, Bourantas CV, Mi QY, Yu YH, Li JJ, Zhao SL, Tian NL and Chen SL: Berberine attenuates adverse left ventricular remodeling and cardiac dysfunction after acute myocardial infarction in rats: Role of autophagy. Clin Exp Pharmacol Physiol 41: 995-1002, 2014.

26. Lin C, Liu Z, Lu Y, Yao Y, Zhang Y, Ma Z, Kuai M, Sun X, Sun S, Jing Y, et al: Cardioprotective effect of Salvianolic acid B on acute myocardial infarction by promoting autophagy and neovascularization and inhibiting apoptosis. J Pharm Pharmacol 68: 941-952, 2016.

27. Rogov V, Dotsch V, Johansen T and Kirkin V: Interactions between autophagy receptors and ubiquitin-like proteins form the molecular basis for selective autophagy. Mol Cell 53: 167-178, 2014.

28. Gottlieb RA: Autophagy in health and disease. Academic Press, London, Waltham, MA, 2013.

29. Lehman JJ, Barger PM, Kovacs A, Saffitz JE, Medeiros DM and Kelly DP: Peroxisome proliferator-activated receptor gamma coactivator-1 promotes cardiac mitochondrial biogenesis. J Clin Invest 106: 847-856, 2000.

30. Olson BL, Hock MB, Ekholm-Reed S, Wohlschlegel JA, Dev KK, Kralli A and Reed SI: SCFCdc4 acts antagonistically to the PGC-1alpha transcriptional coactivator by targeting it for ubiquitin-mediated proteolysis. Genes Dev 22: 252-264, 2008.

31. Xu R, Hu Q, Ma Q, Liu C and Wang G: The protease Omi regulates mitochondrial biogenesis through the GSK3 $\beta /$ PGC- $1 \alpha$ pathway. Cell Death Dis 5: e1373, 2014.

32. Loboda A, Damulewicz M, Pyza E, Jozkowicz A and Dulak J: Role of Nrf2/HO-1 system in development, oxidative stress response and diseases: An evolutionarily conserved mechanism. Cell Mol Life Sci 73: 3221-3247, 2016.

33. Qiao L, Liu Y, Chen X, Xie J, Zhang Y, Yang K, Zhou H, Duan Y, Zheng W and Xie W: A HPLC-MS/MS method for determination of 6"'-feruloylspinosin in rat plasma and tissues: Pharmacokinetics and tissue distribution study. J Pharm Biomed Anal 121: 77-83, 2016

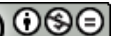

This work is licensed under a Creative Commons Attribution-NonCommercial-NoDerivatives 4.0 International (CC BY-NC-ND 4.0) License. 